\title{
La participación masculina en el cuidado de los hijos en la nueva familia urbana española*
}

\author{
Gerardo M eil Landwerlin \\ Universidad Autónoma de M adrid. \\ M adrid. Spain
}

\section{Resumen}

En este trabajo se analiza la evolución de la participación del hombre en las tareas de atención y cuidado de los hijos en las nuevas familias urbanas durante la última década, así como los factores de los que depende dicha participación. Los resultados que de este estudio se obtienen evidencian un cambio apreciable pero no muy elevado durante la última déca da, así como la necesidad de distinguir entre tipos de actividades o tareas relacionadas con la atención y el cuidado de los hijos, pues las pautas de división según el género de estas actividades depende del tipo de tareas de que se trate. La participación del hombre en las tareas matinales están exclusivamente condicionadas por el estatus laboral de la mujer, mientras que las demás tareas, ya sean cotidianas o más o menos esporádicas, están más condicionadas por la sobrecarga relativa de las familias y el nivel educativo de los padres. EI juego con los niños, por el contrario, está únicamente condicionado por la edad de éstos.

Palabras clave: cuidado niños, actividad doméstica masculina, familias urbanas.

Abstract. M ale participacipation in child care in the Spanish new urban family

This piece of work analizes the evolution of male participation in child care in new urban families during the last decades. It also heats the main factors of the male participation in domestic work. It shows how labour status of women influences domestic male participation.

Key words: child care, domestic male activity, urban families.

* Esta investigación ha sido posible gracias a la financiación recibida del M inisterio de Educación y Ciencia. Proyectos de investigación financiados por la D G YCIT, proyecto PB-93-0239. El autor agradece no sólo la financiación recibida por el M EC, sino también a los investigadores L.E. Alonso y L. Pérez O rtiz, miembros del equipo de investigación y sin cuya preciada colaboración y discusión no habría sido posible esta investigación. 


\section{Sumario}

Las pautas de atención y cuidado de los hijos en las nuevas familias urbanas

Factores explicativos de la participación masculina
O peracionalización de las hipótesis explicativas y metodología de análisis de los datos

Resultados

Resumen y conclusiones

Bibliografía

En las nuevas ciudades que han ido creciendo al rededor de los grandes núcleos urbanos como M adrid o Barcelona, al igual que en los nuevos barrios de las ciudades más viejas, es cada vez más frecuente encontrar a padres con sus hijos pequeños, tanto los sábados como al final de la tarde en los días soleados de la semana, en los parques o avenidas ataviados con triciclo, bicicleta, balón y otros enseres además del periódico. Tampoco ya es infrecuente encontrar varias parejas paseando juntas en las que los padres charlando van empujando la silla con el niño, mientras las mujeres caminan detrás libres de unos atributos que les eran antes atribuidos por «natural eza» (social). Esta imagen externa de la definición de los roles domésticos de hombres y mujeres cada vez menos exótica y singular evidencia el cambio que en las pautas de división del trabajo doméstico se está registrando en las nuevas familias urbanas españolas.

Para analizar este cambio, su magnitud y los factores de los que depende la desdiferenciación de los roles parentales en las nuevas familias urbanas españolas, un equipo de investigación dirigido por el autor realizó una encuesta a jóvenes familias de la corona metropolitana de $M$ adrid, en la que se entrevistó a mujeres de familias completas con al menos un hijo menor de trece años ${ }^{1}$. Los datos obtenidos se compararán con los resultados correspondientes a M adrid de la encuesta diseñada por $\mathrm{M}$ a Ángeles Durán para el C entro de Investigaciones Sociológicas (estudio 1433) sobre desigualdad doméstica y familiar, realizada en $1984^{2}$.

1. Esta encuesta se realizó en junio de 1995 en las ciudades de la corona metropolitana de $M$ adrid. La unidad muestral elegida fueron familias formadas por un hombre y una mujer (independientemente de su vínculo matrimonial) con al menos un hijo menor de trece años, siendo la persona entrevistada mediante cuestionario cerrado la madre. Esta limitación en la selección de las unidades muestrales venía dada por la voluntad de analizar las familias con hijos a cargo en las fases iniciales y centrales de sus biografías familiares y se optó por entrevistar a las madres para poder comparar los datos obtenidos con los datos procedentes del estudio núm. 1433 del Centro de Investigaciones Sociológicas, correspondientes al año 1984. El procedimiento de muestreo fue estratificado por conglomerados de forma proporcional en primera etapa (municipios) y segunda etapa (secciones censales) y afijación proporcional por cuotas. El tamaño de la muestra es de 622 entrevistas.

2. La encuesta del CIS se realizó a «amas de casa» mayores de dieciocho años y a efectos comparativos se han tomado los datos (suministrados por el banco de datos del CIS) correspondientes a la Comunidad de M adrid, no considerando los casos en que «no procede» (no tiene hijos, no se realiza la tarea). El calificativo de «ama de casa» se refiere no sólo a la definición 


\section{Las pautas de atención y cuidado de los hijos en las nuevas familias urbanas}

Antes de describir cuales son las pautas de atención y cuidado de los hijos es preciso caracterizar sociológicamente a las familias entrevistadas. Las familias entrevistadas ( $n=622$ ) se caracterizan fundamentalmente por ser jóvenes, al ser la edad media de la mujer 34,7 años (siendo la mediana 35 y la moda 33 y 36 años), y se encuentran en su mayoría en las primeras fases del ciclo familiar (en el 68\% de los casos el hijo mayor tiene menos de trece años). Estas familias son también relativamente reducidas (el número medio de hijos es de 1,96 y sólo un 19\% tienen tres o más hijos) y de dos órdenes generacionales (92\%), como es norma en todas las familias urbanas. Un $38 \%$ de las mujeres trabaja también fuera del hogar, de las que un 77\% lo hace por cuenta ajena y un $32 \%$ lo hace de forma temporal u ocasional. D adas estas características es razonable considerar que representan a las «nuevas familias urbanas» españolas, entendiendo por nuevas el hecho de que sociológicamente representan la realidad social y cultural de la nueva España surgida tras las profundas transformaciones conocidas durante las últimas décadas. Al ser familias jóvenes y residentes en municipios culturalmente integrados en un área metropolitana como $\mathrm{M}$ adrid con poca influencia de una tradición local específica, consideramos que son representativas de las tendencias que presenta el cambio familiar en España.

En estas familias, como sucede también entre las familias tradicionales, la participación de los padres en la atención y el cuidado de los hijos es, del conjunto de tareas familiares típicas, aquella en la que mayor grado de implicación masculina se registra. Así, mientras la preparación de la comida sigue realizándose exclusivamente por la mujer en dos de cada tres hogares (65\%), la atención a los hijos durante las comidas está más compartida, pues la proporción de los hogares en los que lo realiza exclusivamente la madre es del 56\%

como inactivas, en terminología EPA, sino también a las mujeres activas (ocupadas y desemplea das). Al comparar los datos de una y otra encuesta pueden esperarse dos tipos de sesgos, uno referido a la unidad de análisis (familias) y otro de tipo geográfico. En relación al primer tipo es de esperar un sesgo derivado de la inclusión como unidades muestrales de familias en las que los hijos son mayores de trece años, pero es de suponer que buena parte de las respuestas a las preguntas sobre las distintas tareas de atención y cuidado de los hijos proporcionadas en estos casos se recojan bajo la opción «no procede/no se hace», pues estas tareas son específicas de atención y cuidado a menores no adolescentes. El sesgo de carácter geográfico vendría dado por la inclusión en la muestra del CIS de encuestadas residentes en la zona rural de la Comunidad Autónoma y en el municipio de M adrid, y no sólo dela corona metropolitana. con pautas de respuesta posiblemente diferentes. Pero el posible sesgo en sentido tradicional de las respuestas proporcionadas en la zona rural de la Comunidad podría verse compensado con otro posible sesgo en sentido más participativo en las respuestas de las encuestadas residentes en el municipio de $M$ adrid. Por todo ello, consideramos que no existen razones de peso suficiente para invalidar la comparación de unos datos con otros, siempre que se tomen como indicadores de cambio pautas de respuesta claramente diferentes. 
(véase tabla 1 y tabla 5). Por otro lado, mientras que hacer las camas y recoger las habitaciones es competencia exclusiva de las mujeres en un $57 \%$ de los

Tabla 1. Evolución de la participación del padre en la atención y el cuidado de sus hijos, 1984-1995.

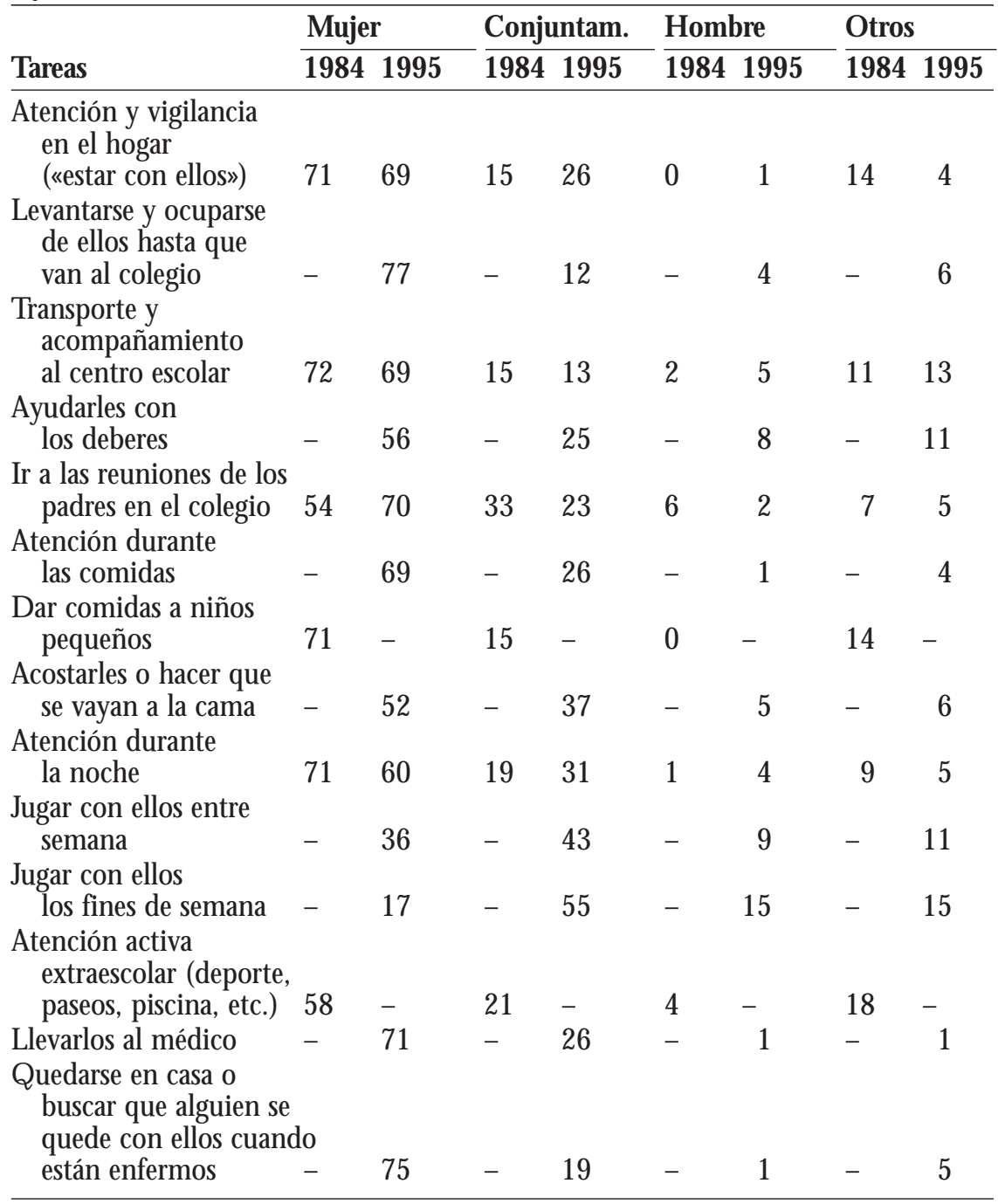

Fuente: Los datos de 1984 corresponden a los resultados para la Comunidad Autónoma de M adrid de la encuesta 1431 del CIS. Los datos correspondientes a 1995 proceden de la «encuesta a nuevas familias en nuevos municipios» realizada por el equipo de investigación dirigido por G erardo M eil y formado por los profesores Luis E. Alonso y Lourdes Pérez (U AM ), con la colaboración de los estudiantes de 40 y 5은 curso de la especialidad de Sociología Económica de la licenciatura en Economía de la U AM . 
hogares, el acostar a los hijos o hacer que éstos vayan a la cama así como el atenderles durante la noche, cuando es preciso, recae en exclusiva en las mujeres en un 410 48\% de los hogares. La atención en general a los hijos se realiza conjuntamente por ambos padres en uno de cada tres hogares (34\%), mientras que la limpieza de la casa es una tarea compartida sólo en una de cada cinco (19\%) familias. Esta mayor participación del hombre en las tareas domésticas relacionadas con los hijos es típica de las nuevas familias y está ampliamente documentada, tanto en España (D urán, 1988), como en otros países occidentales (O akley, 1975; K ellerhals y otros, 1982; Bird y Scruggs, 1984; Koopmann-Boyden y Abbott, 1985; H iller y Philliber, 1986; Rexroat y Shehan, 1987; Keddi y Seidenspinner, 1991).

La atención y el cuidado de los hijos es una actividad familiar multifacética que engloba muchos tipos de tareas diferentes, en las que el hombre no participa de igual manera. Para medir más adecuadamente esta participación hemos incluido una lista de doce tipos de actividades distintas con los hijos, recogidas en la tabla 1, que si bien en conjunto no miden todas las limitaciones a la disponibilidad del tiempo que su presencia implica para la organización del tiempo, mayormente, de las mujeres, sí hace más justicia a la diversidad de tareas a realizar con los hijos que las consideradas habitualmente en otros estudios. La forma más típica y tradicional de participación de los padres, una vez que éstos decidieron acortar las distancias con sus hijos, ha sido, y continúa siéndolo, en las actividades lúdicas, menos costosas y más gratificantes que el resto. Esto es especialmente cierto en el juego durante el fin de semana, como puede comprobarse en dicha tabla 1. D urante el fin de semana, las actividades lúdicas con los hijos conjuntamente realizadas en el marco del ocio familiar son la pauta claramente dominante y entre semana, aunque menos frecuente, también es mayoritaria. Ú nicamente en las familias con hijos más mayores se produce una mayor polarización hacia una mayor implicación exclusiva de la madre o del padre en el juego con los hijos ${ }^{3}$. Esta pauta parece guardar relación con el sexo de los hijos y las distintas necesidades de niños y niñas a distintas edades. En cualquier caso, en las fases más tempranas del ciclo familiar no se observa una discriminación de los padres en sus relaciones con las hijas; cuando los padres juegan con sus hijos pequeños lo hacen independientemente del sexo. Por otra parte, esta mayor participación conjunta de los padres en el juego de los hijos frente a otras tareas menos gratificantes como ir a las reuniones de padres en el colegio o su cuidado y atención cotidiana estambién común en otros países, como Francia (M inistère des Affaires Sociaux, 1988), Alemania (Keddi y Seidenspinner, 1991; N ave-H erz, 1989) o Suiza (Kellerhals y otros, 1982).

3. Así, en las familias en las que el hijo mayor tiene menos de tres años, cuando no hay hijas, un $74 \%$ de las encuestadas declaran que juegan a medias los fines de semana con sus hijos, frente a un $68 \%$ cuando hay hijas. En las familias en las que el hijo mayor tiene más de dieciocho años, con el (los) menores de trece años juegan ambos padres en el $57 \%$ de los casos si no hay hijas y en el $45 \%$ de los casos si hay hijas. Aunque estos indicadores no son muy precisos, apuntan en el sentido indicado. 
Por otro lado, también existen diferencias marcadas según la hora a la que deben realizarse las tareas. El hombre tiende a participar más en tareas que se realizan típicamente por las tardes y/o noches, mientras que su implicación en las tareas matinales como levantarles, ocuparse de ellos hasta que van al colegio y llevarles o acompañarles al colegio está mayormente en manos de las madres, incluso cuando éstas trabajan (una de cada tres encuestadas), aunque en este caso existe un grado de participación al go mayor. Así, tres de cada cuatro mujeres encuestadas levantan y preparan a sus hijos para ir al colegio y una proporción similar los lleva o acompaña al colegio, mientras que el padre de forma habitual solamente colabora en un 16 a 18\% de los casos. N o obstante, en la preparación del desayuno parece que existe algo más de colaboración masculina, pues en un $28 \%$ de las familias encuestadas el desayuno lo preparan a medias o mayormente el padre. Sin descartar la influencia sexista, esta menor participación matinal de los padres debe guardar relación con los diferentes ritmos temporales de cada una de las partes implicadas y de las restricciones temporales impuestas por la relación laboral, pues de lo contrario no se explica que padres que participan activamente en la realización de tareas tradicionalmente consideradas femeninas (como limpiar, cocinar o hacer camas) presenten pautas tradicionales en esta dimensión de la actividad familiar.

La participación en tareas desempeñadas por la tarde-noche es, por el contrario, mucho mayor. Así, el acostarles o hacer que se acuesten es una tarea compartida por más de un tercio de las familias encuestadas (37\%) y atenderles durante la noche, cuando es preciso, también es compartido por una proporción similar (31\%). La atención durante las comidas está también menos compartida, pero en buena medida porque una parte importante de los padres no come regularmente en casa (M inisterio de Asuntos Sociales, 1994).

La participación en tareas educativas, si se juzga a partir de las pautas vigentes en la asistencia a las reuniones de padres en el colegio, donde puede suponerse que asiste el cónyuge que supervisa más directamente la educación de los hijos, continúa siendo bastante tradicional, pues la participación indiferenciada en esta tarea (que únicamente hay que realizar una o dos veces al año) apenas si se eleva a una de cada cuatro familias (23\% más un $2 \%$ en el que lo hace el hombre habitualmente), cuando los datos proporcionadados para otros países son apreciablemente mayores, 29\% en Francia (M AS, 1988) y $34 \%$ en Alemania (K eddi y Seiddenspinner, 1991). Si se juzga, por el contrario, a partir de la ayuda prestada a los hijos para realizar los deberes, la imagen que presentan las nuevas familias es mucho menos tradicional. Así, en las familias con hijos en edad escolar o adolescentes, la participación masculina activa (en exclusiva 0 de forma alternada con la mujer) se el eva entre el 44 y el $50 \% 4$.

4. Así, la ayuda a los hijos en los deberes por parte del padre en la mayoría de los casos pasa del $9 \%$ cuando el hijo mayor tiene de 6 a 12 años, al 14\% cuando tiene de 13 a 17 años y al $24 \%$ cuando tiene más de 18 años. La ayuda «a medias» pasa del 37 al 36 y al 20\% respectivamente. 
En la atención y el cuidado en caso de enfermedad, aún estando mayormente en manos de las madres, la implicación del marido, a juzgar por las afirmaciones de las encuestadas, es bastante mayor de lo que cabría esperar a partir de la observación de las consultas de pediatría en los centros de salud. Según éstas, en uno de cada cuatro casos la visita al médico se hace bien conjunta mente, bien según una pauta indiferenciada, y esta misma pauta se aplica en una de cada cinco familias para buscar soluciones para que al guien se quede con ellos en caso de enfermedad. El hecho de que un tercio de las encuestadas que afirman no tener una pauta tradicional de cuidado en caso de enfermedad no realice tareas laborales extradomésticas Ileva a sospechar que la participación masculina está sobrevalorada por las propias encuestadas.

En conjunto, la imagen global que se obtiene de las nuevas familias es moderadamente participativa: en un 15\% de las familias encuestadas el hombre participa de forma activa ( «a medias» según la opción de respuesta propuesta) en ocho de las diez tareas consideradas (excluido el juego) y son una de cada cuatro $(25 \%)$ las familias en las que el hombre participa activamenteen siete tareas. La comparación con los datos obtenidos de la encuesta del CIS para 1984 y referidos también a M adrid evidencia, como puede observarse en la misma tabla 1, un cambio apreciable, aunque no especialmente elevado, durante la última década en dirección hacia una menor realización exclusiva de la mujer de la mayor parte de las tareas relacionadas con la atención y el cuidado de los hijos y que se traduce no en una derivación hacia otras personas de estas tareas, sino en una mayor participación masculina en las mismas. En las tareas relacionadas con la educación, sin embargo, se registra una pauta inversa: en el acompañamiento al centro escolar apenas se producen cambios significativos entre 1984 y 1995, y en la asistencia a las reuniones de padres se registra una tendencia muy marcada hacia una vuelta a pautas tradicionales. No obstante, esta tendencia no puede interpretarse, como acaba de verse, como una tendencia al descompromiso de los padres con la educación de sus hijos y, por tanto, como un retorno a pautas de tipo tradicional. Por otra parte, si consideramos que la «realización de tareas extraescolares (deporte, piscina, paseos, etc.)» es equivalente a la tarea de «jugar», esto es, que las encuestadas han entendido en ambos casos más o menos el mismo tipo de actividades, podemos observar cómo el aumento registrado de la participación del hombre durante la última década se ha centrado no en la actividad lúdica con los hijos o en las tareas matinales, sino fundamentalmente en tareas que se desarrollan por la tardenoche, esto es, en la mayor parte de los casos durante el tiempo no dedicado al trabajo.

El modelo de cambio que se perfila en esta comparación apunta así en dirección hacia una mayor participación en tareas habitualmente consideradas más arduas y menos gratificantes, más que a una densificación de las relaciones padre-hijos en las actividades de ocio. Esta característica del cambio familiar puede considerarse paralela al importante aumento de la proporción de familias en las que el hombre participa en tareas domésticas tradicionalmente consideradas femeninas (cocinar, limpiar, recoger, etc.) (véase tabla 5). 
D e hecho, el mayor grado de cambio en las pautas de división del trabajo doméstico en la pasada década no se ha producido en la atención y el cuidado de los hijos, sino en las tareas domésticas propiamente dichas. Así, mientras la participación masculina en la compra diaria se ha triplicado durante la última década (medido a partir de la proporción de respuestas que señalan que la tarea se realiza conjuntamente), se ha multiplicado entre cuatro y ocho veces en las distintas tareas que se realizan en la cocina y entre cuatro y seis o más en las distintas tareas de limpieza, la participación en las distintas tareas de cuidado de los hijos no ha llegado a duplicarse. Por tanto, este aumento de la participación masculina en la atención y el cuidado de los hijos se enmarca en un proceso más amplio de desdiferenciación de los roles parentales y domésticos en el seno de las familias urbanas españolas.

\section{Factores explicativos de la participación masculina}

Para explicar la participación masculina en las tareas relacionadas con la atención y el cuidado de los hijos no existe un modelo explicativo diferenciado del resto de las tareas domésticas, dado que la mayor parte de los estudios considera el cuidado de los niños como una tarea doméstica más. Ahora bien, la participación en esta tarea tiene unas características específicas que no tienen el resto de tareas domésticas. Así, habitual mente son consideradas menos repetitivas, más variadas y más gratificantes que estas tareas domésticas. Por otro lado, culturalmente existe una mayor presión en dirección hacia una mayor participación y compromiso del padre, todo lo cual redunda de hecho en una sustancial mayor participación masculina en las tareas relacionadas con la crianza de los niños que en la realización de tareas domésticas.

La característica socioeconómica que en la mayor parte de los estudios empíricos (fundamental mente norteamericanos) se manifiesta como más determinante de la participación masculina es el estatus laboral de la mujer. Si la mujer dispone de un trabajo extradoméstico se registra, independientemente de cualquier otra característica, una mayor participación en tareas directamente relacionadas con el cuidado de los hijos, tales como vigilancia, asistencia a reuniones de padres o más genéricamente atención, que en el caso de que la mujer se dedique en exclusiva a las tareas domésticas (Kellerhals y colbs., 1982; M aret y Finlay, 1984; H iller y Philliber, 1986; H egner y Lackermann, 1989; Keddi y Seidenspinner, 1991). Algunos estudios evidencian, sin embargo, que la mayor participación del hombre en el cuidado de sus hijos no depende tanto de si la mujer dispone o no de un trabajo extradoméstico, como del estatus profesional que ha logrado, dándose la mayor participación en el caso en el que la mujer prosigue una carrera profesional (esto es, las dual-career families) (Bird y Scruggs, 1984; Barnett y Baruch, 1987), pero no en los demás casos. El que los hombres participen en el cuidado y en la atención de los hijos no significa, sin embargo, como han tratado de evidenciar algunos estudios (Leslie, Anderson y Branson, 1991), que sean ellos quienes asuman la responsabilidad integral sobre los mismos, entendida ésta como algo más que la resolución de tareas específicas. 
M ás allá del estatus laboral, y en al gunos casos del estatus profesional, no se ha medido la influencia de otras variables (ingresos o nivel de estudios) que midan los recursos que la mujer puede hacer valer fuera de la relación conyugal y que pueda utilizar en el seno de ésta para negociar el contenido de los roles conyugales, tal como postula la teoría de los recursos y como se ha tratado de contrastar en los estudios sobre distribución de tareas domésticas. Salvo en el citado estudio de Bird y Scruggs, basado en una muestra de familias de clase media, y en el que sí se evidencia una mayor participación en las familias de doble carrera, no parece que la teoría de los recursos pueda explicar la distinta implicación masculina en el cuidado de los hijos. De hecho, algunos estudios han analizado la influencia del nivel de estudios y/o del nivel profesional del hombre, no encontrando ningún efecto sistemático de estas variables, ni de carácter negativo, como postula la teoría de los recursos, ni de carácter positivo, como cabría esperar a partir de la hi pótesis ideológica (según la cual la segregación de los roles conyugales disminuye con el nivel educativo-cultural de los cónyuges) (Robinson, 1977; Covermann y Sheley, 1986; Barnett y Baruch, 1987; K eddi y Seidenspinner, 1991). O tra conclusión que se desprende de estos estudios es que no parece existir una pauta diferenciada de participación en las tareas de cuidado de los hijos en los distintos estratos sociales, aunque los estilos de socialización sí puedan y de hecho varíen apreciablemente de un estrato social a otro (Kellerhals y otros, 1993; M AS, 1994). Los estilos de socialización no estarían, por tanto, directamente relacionados con el grado de implicación del padre en la atención y el cuidado de los hijos.

La ideología de rol, en los pocos casos en los que se ha anal izado su efecto sobre las pautas de cuidado de los hijos, no se ha evidenciado como un factor explicativo significativo. En el estudio de Bird y Scruggs (1984), cuando el hombre apoyaba explícitamente las aspiraciones de la mujer para desarrollar su carrera profesional, se registraba una mayor participación masculina, pero en las familias en las que el trabajo extradoméstico de la mujer no implicaba una profesión absorbente, los valores no contribuían a explicar las diferentes pautas. Así, en el estudio de H iller y Philliber (1986) mientras el $84 \%$ de los encuestados, ya fuera la mujer o su cónyuge, opinaban que el cuidado de los niños debía compartirse, la proporción en la que las distintas tareas relacionadas con el cuidado y la atención de los niños se hacía de forma compartida o mayormente por el hombre se elevaba como máximo al 50\%, con un abanico que comenzaba con el $16 \%$ de participación en las tareas matinales y alcanzaba el 54\% con la ayuda con los deberes. La única variable estadísticamente significativa que en dicho estudio explicaba parte de la varianza en las pautas de atención y cuidado de los hijos era el estatus laboral de la mujer. Por otro lado, las respuestas dadas por los 10.000 encuestados/as alemanes analizados por Keddi y Seidenspinner (1991) evidencian que entre las parejas cohabitantes (supuestamente más igualitaristas) se da una menor realización exclusiva o mayoritaria de estas tareas por parte de la mujer que entre los encuestados casados. D ado que estas diferencias no se controlan por la influencia de otras 
variables, es posible, sin embargo (como sucede en estudios sobre la división de tareas domésticas), que estas diferencias sean espúreas o recojan la incidencia de otras variables.

Los estudios que han anal izado la influencia de la sobrecarga relativa de la familia, a partir del análisis diferencial del tiempo disponible por ambos cónyuges y la cantidad de tareas a resolver (medida a través del número y/o de la edad de los hijos), tampoco han abundado y los datos disponibles no permiten afirmaciones concluyentes. Según algunos estudios sobre tiempos destinados a las distintas tareas familiares y domésticas, las mujeres que trabajan afirman dedicar menos tiempo al cuidado de los hijos que aquellas que no tra bajan, por lo que la participación relativa del hombre aumenta, pero no el tiempo real dedicado por el hombre (Blair y Lichter, 1991; Leslie, Anderson y Branson, 1991). En otros estudios, por el contrario, la disminución del tiempo dedicado por la mujer se compensa parcialmente por un aumento del tiempo dedicado por los hombres a cuidar sus hijos (H egner y Lackermann, 1989), tiempo que aumenta con el número de horas dedicado por la mujer al trabajo (Barnett y Baruch, 1987). Coverman y Sheley (1986) han encontrado una relación negativa entre el tiempo dedicado por el hombre al trabajo y el tiempo que dedica a sus hijos, aunque la lineal idad de la relación desaparece entre 1965 y 1975, al tiempo que crece la participación cuanto menor es la edad, particularmente cuando los hijos son menores de tres años, relación que también esta documentada por Rexroat y Shehan (1987).

Si se considera la tenencia de un trabajo extradoméstico como un condicionante de la disponibilidad temporal de la mujer, en lugar de interpretarlo como un recurso o como expresión de una ideología de rol no tradicional, la participación del hombre en el cuidado de los hijos guardaría relación más con necesidades e imperativos familiares y disponibilidades temporales de los cónyuges que con procesos más globales de cambio sociocultural en dirección hacia una «nueva paternidad» más responsable. La contrastación de esta hipótesis no ha sido, sin embargo, hasta el presente explícitamente planteada y ana lizada.

En lo que sigue se van a analizar a partir de los datos de la «encuesta a nuevas familias en nuevos municipios» los factores de los que depende la participación masculina en las tareas de atención y cuidado delos niños. Las hipótesis explicativas que quieren contrastarse son las habitualmente consideradas en la literatura sobre la segregación de los roles familiares ${ }^{5}$, concretamente:

1. Sobre la base de una imagen social en la que el hombre debe participar igualitariamente en las tareas de atención y cuidado de los niños, su participación guardaría relación primordialmente con las limitaciones temporales de los cónyuges así como con la sobrecarga relativa de la familia, en el sen-

5. Una discusión más amplia de estas hipótesis explicativas puede encontrarse en M eil (1995), C overman (1985), England y Farkas (1986) o H öpflinger y Charles (1990). 
tido de que cuanto mayor es la edad de los hijos, mayor es su autonomía personal, disminuyendo, por tanto, la carga de determinadas tareas a realizar (atención durante las comidas, atención durante la noche o preparación matinal para ir al colegio) y con ello la participación de los padres en este tipo de tareas ${ }^{6}$. D esde este punto de vista, la participación de los padres debería ser mayor cuando se diera alguna de las siguientes situaciones individualmente consideradas o de forma conjunta: presencia de neonatos, niños pequeños, número de niños y/o número de horas trabajadas fuera del hogar o tipo de trabajo extradoméstico de la mujer. El número de horas trabajadas por el hombre y, más concretamente, su hora de llegada al hogar operaría en sentido inverso.

2. La hipótesis de que la participación masculina responde a la existencia de una nueva concepción de la paternidad más responsable entre los hombres no puede analizarse, pues la encuesta solamente se ha realizado a madres de al menos un hijo menor de trece años, no disponiéndose, por tanto, de datos sobre actitudes de los hombres. Una vía indirecta para analizar la relación entre los cambios en las pautas de participación del hombre en las tareas de atención y cuidado de los niños y los cambios en la definición de los roles parentales podría ser a través del análisis de la influencia de la ideología de rol maternal con la que se identifican las madres, en el sentido de cuanto menos tradicional es la definición del rol maternal (fundamental mente medida a través del tipo de respuestas dadas al conflicto potencial entre maternidad y trabajo extradoméstico) mayor es la participación masculina. Al respecto es preciso tener presente no sólo la tradicional censura entre actitudes y comportamientos, sino también que una concepción igualitarista de los roles parentales por parte de la madre no tiene por qué traducirse necesariamente en una capacidad efectiva para imponer pautas de comportamiento en el hombre acorde con dicha concepción. Esta capacidad para lograr establecer un determinado orden familiar puede depender más de otros condicionantes de la relación conyugal, que de la concepción de la mujer sobre cómo debería funcionar idealmente la organización familiar.

3. La participación masculina puede depender de hecho más, tal como se sostiene por la teoría de los recursos, de los recursos que la mujer pueda hacer valer en el mercado de trabajo y que redundan en un aumento del poder de

6. En esta encuesta la participación en las tareas domésticas y familiares ha sido medida mediante una escala «cualitativa» de evaluación de quién realiza la tarea propuesta (mujer sólo, mujer mayormente, a medias, hombre sólo o mayormente, entre todos los de la familia y otras respuestas). D ado que, por tanto, no se mide el tiempo destinado a las mismas, en realidad no puede medirse el grado de participación diferencial de los cónyuges. A hora bien, dado que la mayoría de las encuestadas, independientemente de la edad del hijo, respondieron si lo hacía la mujer, el hombre o a medias y no en la opción «no procede», puede considerarse que la definición por defecto es «lo real iza la mujer» y que lo que mide la escala es la variación en la participación masculina. 
negociación del contenido de los roles en el seno de la relación conyugal, que de su propia concepción sobre si y cómo debería participar el padre en las tareas de atención y cuidado de los hijos. D e acuerdo con esta hipótesis, la participación masculina crecería no sólo en función de si la mujer dispone de un trabajo extradoméstico o no, sino de la remuneración en prestigio o dinero que con ello obtiene.

4. Por último, en una simplificación de la tesis de Bott (1971), según la cual la segregación de los roles conyugales depende de la conectividad de la red de relaciones sociales de las familias, y dado que en la encuesta se recogían también distintas dimensiones de la solidaridad interfamiliar, se va a contrastar la hipótesis de que la participación masculina se encuentra en relación inversa con la ayuda recibida por parte de la parentela en las tareas de atención y cuidado de los niños.

\section{O peracionalización de las hipótesis explicativas y metodología de análisis de los datos}

Para medir la hipótesis de la sobrecarga familiar relativa se utilizará como indicador de la disponibilidad de tiempo del hombre la hora de su llegada a casa (antes de las 6 de la tarde, entre 6 y 7, entre 7 y 8 y más tarde de las 8, considerando como valores perdidos quienes declaraban que no tenía horario fijo), bajo el supuesto de que cuando los hijos están escolarizados es más relevantela hora de llegada al domicilio que el número real de horas trabajadas. La disponibilidad de tiempo de la mujer se ha medido, por un lado, a través de una serie de variables ficticias (dummies) que recogen la duración media de la jornada semanal (menos de 10 horas, de 11 a 30, de 31 a 40 y más de 40), tomando como referencia el que la mujer no dispone de trabajo extradoméstico. Por otro lado, también se ha distinguido mediante otra serie de variables ficticias entre trabajo continuo y trabajo no continuo (temporal u ocasional), tomando como base igualmente la ausencia de una relación laboral extradoméstica. Para medir la sobrecarga relativa se consideró la edad del hijo menor y el número de hijos.

En la operacionalización de la ideología de rol maternal los indicadores más discriminantes son aquellos que indagan sobre las normas que deben regir la reorganización familiar cuando se produce el nacimiento de un hijo. Concretamente, se preguntó a las encuestadas que mostraran su grado de acuerdo o desacuerdo con la norma «la mujer debería abandonar su trabajo si ello interfiere en el cumplimiento de sus obligaciones como madre y esposa». Así mismo se pidió a las encuestadas que señalaran, en el caso de que pudieran realmente elegir, ¿quién debería mantener económicamente a la familia cuando hay niños y éstos todavía no pueden ir al colegio? (las opciones de respuesta eran hombre, ambos y otras, opción ésta considerada como valor perdido).

Para medir los recursos que los cónyuges pueden hacer valer en el seno de la relación conyugal, se pidió a las encuestadas que indicaran su nivel educativo así como el de su cónyuge en un escala de 1 (no tiene estudios terminados) a 
5 puntos (estudios universitarios concluidos), procediéndose a continuación a medir la diferencia entre el nivel educativo de la mujer y del hombre, como medida de diferencia relativa de los cónyuges. En qué medida el nivel educativo es un recurso o no que el hombre o la mujer puede utilizar para definir los roles conyugales o es un indicador de la ideología de rol es una cuestión ampliamente debatida. En la «encuesta a nuevas familias en nuevos municipios», de hecho, no existe relación estadísticamente significativa entre las distintas normas propuestas para medir la ideología del rol maternal y conyugal de la mujer y su nivel educativo ${ }^{7}$. Para medir los ingresos se pidió a las encuestadas indicaran en una tarjeta en la que se incluían nueve tramos de ingresos netos mensuales, en qué categoría se encontraban sus ingresos y los de su cónyuge. Para medir la diferencia de recursos se procedió posteriormente a medir la diferencia entre ambas variables, pero dadas las dudas existentes sobre la veracidad de la respuesta se pidió a las encuestadas indicaran también si sus ingresos eran menores (1) o mayores (4) que los del marido en una escala de 4 puntos. También se preguntó la profesión de ambos cónyuges según una escala de 5 puntos (1, empresarios, directivos, profesionales independientes; 2 , cuadros, técnico y funcionarios de gestión; 3 , administrativos y trabajadores cualificados; 4, trabajadores no cualificados; 5, inactivos).

El análisis de la incidencia de la solidaridad interfamiliar se realizará a partir de las respuestas dadas a la pregunta sobre la frecuencia de la ayuda recibida por parte de los padres y suegros en la atención y el cuidado de los niños, señalándose como opciones «con mucha frecuencia», «con frecuencia», «de vez en cuando», «en casos muy particulares» y «no procede» (considerado como valor perdido).

Por último, dada la importancia que el ciclo familiar tiene en el análisis de la dinámica familiar y su posible incidencia en la realización de determinadas tareas con los hijos, el ciclo familiar se operacionalizó, por un lado, a partir de la edad del hijo mayor distinguiendo las fases 0 a 2 años, 3 a 5 años, 6 a 13 años, 13 a 18 años y más de 18 años y tratadas como variables ficticias, tomando como referencia la primera fase. Por otro lado, y en los casos en los que así lo requería la hipótesis a contrastar, se consideró la edad del hijo menor.

D ado que las doce tareas consideradas para medir las pautas de atención y cuidado de los niños no son homogéneas en términos de frecuencia de realización en el tiempo, tiempo que demandan para su cumplimiento y dependencia que generan, en lugar de construir una escala aditiva simple de atención

7. Además de las proposiciones mencionadas en el texto, la ideología de rol se trató de medir también a través de los siguientes indicadores: «cuando hay niños pequeños, el tipo de trabajo ideal para una mujer es el trabajo de media jornada»; «a mujer casada y con hijos debería poder planificar su futuro profesional de la misma forma en que lo hace su marido», «el marido no debería molestarse si, por ejemplo, el trabajo de su mujer exige que pase al gunos días fuera de casa»; «el hombre, cuando es el único que mantiene económicamente a la familia, no debería asumir demasiadas tareas domésticas», «si ambos padres trabajan, el padre debería estar dispuesto a reducir sus compromisos laborales para poder cuidar de los niños y realizar tareas domésticas». 
Tabla 2. Análisis de componentes principales de las tareas de atención y cuidado de los niños.

\begin{tabular}{lcccr}
\hline & Factor 1 & Factor $\mathbf{2}$ & Factor $\mathbf{3}$ & Factor 4 \\
\cline { 2 - 5 } & $\begin{array}{l}\text { Atención } \\
\text { educativo- } \\
\text { sanitaria }\end{array}$ & $\begin{array}{l}\text { Atención } \\
\text { cotidiana }\end{array}$ & $\begin{array}{l}\text { Tareas } \\
\text { matinales }\end{array}$ & Juegos \\
\hline Visita al médico &, 73622 &, 20306 &, 04212 &,- 00176 \\
Asistir a reuniones de padres &, 73191 &,- 06611 &, 24319 &, 04785 \\
Cuidado en caso de enfermedad &, 64073 &, 28796 &, 09568 &,- 01309 \\
Ayuda con deberes &, 45390 &,- 12630 &, 06051 &, 44763 \\
Atención por la noche &, 05079 &, 75470 &, 10111 &,- 02417 \\
Acostarles &,- 00067 &, 72368 &, 01385 &, 14842 \\
Atención y vigilancia en hogar &, 31938 &, 50156 &, 28332 &, 08232 \\
Atención durante las comidas &, 35140 &, 49752 &, 14969 &, 21255 \\
Transporte al colegio &, 17255 &, 02250 &, 87989 &, 05274 \\
Levantarles y ocuparse de ellos &, 13546 &, 28562 &, 81087 &,- 00751 \\
Jugar los fines de semana &,- 10806 &, 09547 &, 01258 &, 83037 \\
Jugar entre semana &, 12632 &, 20888 &, 01782 &, 80128 \\
\hline
\end{tabular}

y cuidado, se procedió a realizar un análisis de componentes principales que lograra identificar grupos de tareas con pautas de resolución homogéneas. El aná-

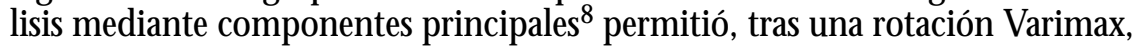
la identificación de cuatro factores que corresponden a cuatro grupos de tareas. El factor 1 corresponde a tareas más o menos esporádicas referidas a la educación (asistencia a reuniones de padres, ayuda con los deberes) y al cuidado en caso de enfermedad (Ilevarlos al médico y quedarse en casa con ellos o encontrar una solución para su cuidado). El factor 2 corresponde, por el contrario, a lo que podríamos denominar «atención cotidiana», que incluye el acostarlos, la atención durante la noche así como durante las comidas y, en general, la atención y vigilancia en el hogar («estar con ellos»). El factor 3 recoge las tareas matinal es, tanto de levantarles y ocuparse de ellos hasta que van al colegio, como el propio hecho de llevarlos al colegio. El factor 4 y último está claramente definido por las tareas lúdicas, esto es, jugar con ellos, tanto entre semana como durante los fines de semana.

I dentificados estos cuatro grupos de tareas de atención y cuidado de los niños se procedió a construir un indicador aditivo simple para cada uno de estos tipos de tareas. Los indicadores de fiabilidad al pha Cronbach para cada uno de los cuatro grupos de tareas resultaron, como era lógico esperar, mayormente satisfactorios (sólo la atención sanitario-educativa tiene un indicador

8. El valor de medida K aiser-M eyer-O Ikin de la adecuación de la muestra es de 0,76, convencionalmente considerado bueno. 
relativamente bajo - 0,58-, en los demás casos se sitúan al rededor de 0,7). Con estos cuatro indicadores resumidos de atención y cuidado de los niños se contrastaron las hipótesis arriba formuladas mediante un análisis de regresión múltiple mediante ajuste por mínimos cuadrados.

\section{Resultados ${ }^{9}$}

En primer lugar se procedió a contrastar cada una de las hipótesis explicativas por separado, para a continuación contrastar el conjunto de variables explicativas con el fin de obtener el mejor ajuste posible. En la tabla 4 se recogen los resultados de contrastar las distintas hipótesis de forma alternativa, esto es, considerando cada vez sólo una de las hipótesis. Las variables que miden la diferencia relativa de recursos entre los cónyuges se han tratado aisladamente de las variables que miden los niveles absolutos de cada cónyuge, debido tanto al problema de multicolinealidad que en caso contrario se produciría, como en razón a la distinta dimensión que recogen (los recursos absolutos miden fundamental mente diferencias de género y de estrato social y los recursos relativos miden diferencias de poder). Por otro lado, aquellas variables para las que no se podía rechazar la hipótesis nula de ausencia de relación se han dejado fuera del modelo de regresión. Como puede verse en dicha tabla, la capacidad explicativa de las distintas hipótesis explicativas depende del tipo de tarea a realizar.

En el juego las únicas variables que tienen cierto grado de capacidad explicativa son la hora de llegada del padre al hogar, en el sentido de que cuanto más tarde llega éste menos juega con los niños, y la profesión de la mujer (cuando trabaja fuera del hogar), en el sentido de que cuanto mayor es su nivel profesional más participa el varón ${ }^{10}$, si bien es preciso destacar que la capacidad explicativa de la escasa variación en las pautas de juego es extremadamente baja (un $1 \%$ en cada caso).

En la atención matinal puede observarse que la participación masculina depende exclusivamente del estatus laboral de la mujer, pero no tanto de si trabaja fuera del hogar o no, como del estatus social que con ello logra. C uanto mayor es la cualificación profesional y los ingresos que con ello logra, mayor es

9. El supuesto de linealidad se ha contrastado para las variables que en principio puede sospecharse no se cumple (educación del hombre y de la mujer) a través de la comparación de los resultados del análisis de varianza de la regresión con dichas variables tratadas como variables continuas y como variables dummy (Lewis-Beck, 1993: 307). El supuesto de no aditividad se contrastó a través de un análisis de varianza en el que se testaron efectos interactivos de segundo orden entre dichas variables, el estatus laboral de la mujer y la fase del ciclo familiar. Los resultados de dichos análisis evidenciaron una relación lineal y aditiva, por tanto, sin efectos interactivos.

10. Recuérdese que en virtud dela construcción del indicador, lo que se mide no es cuánto realiza cada cónyuge de una determinada tarea, sino si el hombre participa más o menos en la resolución del grupo de tareas de las que se trate, independientemente de la frecuencia con que se realicen 0 el tiempo que se le dedique. 
Tabla 3. Coeficientes de regresión parcial sin estandarizar (estandarizados) para las regresiones de los distintos grupos de tareas de atención y cuidado de los hijos, según las distintas hipótesis explicativas. A. Atención matinal; B. Atención cotidiana; C. Atención educativo-sanitaria; D. Juegos.

\begin{tabular}{|c|c|c|c|c|}
\hline H ipótesis explicativas & $\mathbf{A}$ & B & C & D \\
\hline \multicolumn{5}{|c|}{ H ipótesis de los recursos (niveles absolutos) } \\
\hline N ivel educativo del hombre & - & $0,06(0,11)^{*}$ & $0,10(0,20) *$ & - \\
\hline N ivel profesional del hombre & - & - & & - \\
\hline$N$ ivel de ingresos del hombre & - & - & $-0,04(-0,12)^{* *}$ & - \\
\hline N ivel educativo de la mujer & - & $0,06(0,11)^{*}$ & & - \\
\hline N ivel profesional de la mujer & $0,18(0,70)^{*}$ & $0,1(0,16)^{* *}$ & $0,05(0,09)^{*}$ & $0,09(0,12) *$ \\
\hline$N$ ivel de ingresos de la mujer & $0,14(0,27)^{* *}$ & & & \\
\hline Constante & 0,71 & 1,35 & 1,53 & 2,43 \\
\hline R2 ajustada (n) & 0,145 & $0,08(590)$ & $0,04(492)$ & $0,01(558)$ \\
\hline \multicolumn{5}{|c|}{ H ipótesis de los recursos (niveles relativos, hombre-mujer) } \\
\hline Diferencia de nivel de estudios & - & - & $0,08 / 0,14 * *$ & - \\
\hline Diferencia de nivel profesional & - & - & - & $0,06(0,95)^{*}$ \\
\hline Diferencia de nivel de ingresos & $-0,12(-0,27)^{* *}$ & $-0,04(-0,14)^{*}$ & - & \\
\hline $\begin{array}{l}\text { Constante } \\
\text { R2 ajustada (n) }\end{array}$ & 2,02 & 2,25 & $\begin{array}{l}1,69 \\
0\end{array}$ & 2,68 \\
\hline \multirow{2}{*}{\multicolumn{5}{|c|}{$\begin{array}{l}\text { H ipótesis de la sobrecarga } \\
\text { D uración de la jornada laboral (base no trabaja): }\end{array}$}} \\
\hline & & & & \\
\hline M enos de 10 horas & - & - & - & - \\
\hline De 10 a 30 horas & $0,34(0,12)^{* *}$ & - & - & - \\
\hline De 31 a 40 horas & $0,71(0,29)^{* *}$ & $0,48(0,24)^{* *}$ & $0,32(0,18)^{* *}$ & - \\
\hline M ás de 40 horas & $0,77(0,25)^{* *}$ & $0,27(0,10)^{*}$ & $0,30(0,12)^{* *}$ & - \\
\hline N ro. de hijos & $0,79(0,08)^{*}$ & - & - & - \\
\hline \multirow{2}{*}{\multicolumn{5}{|c|}{$\begin{array}{l}\text { Edad del hijo menor } \\
\text { Ciclo familiar (base } 0 \text { a 3) }\end{array}$}} \\
\hline & & & & \\
\hline Edad mayor de 4 a 5 & & & $0,20(0,10)+$ & \\
\hline $\begin{array}{l}\text { Edad mayor de } 6 \text { a } 13 \\
\text { Edad mayor de } 13 \text { a } 18\end{array}$ & & & $0,37(0,23)^{* *}$ & $-0,24(-0,14)^{*}$ \\
\hline & & & $0,29(0,14)^{* *}$ & $-0,26(-0,09)+$ \\
\hline H ora llegada hombre a casa & - & $-0,03(-0,15)^{*}$ & $-0,13(-0,25)^{* *}$ & $-0,10(-0,14) * *$ \\
\hline Constante & 1,21 & & & 2,96 \\
\hline R2 ajustada (n) & $0,12(564)$ & $0,10(333)$ & $0,14(412)$ & $0,03(404)$ \\
\hline \multirow{2}{*}{\multicolumn{5}{|c|}{$\begin{array}{l}\text { H ipótesis de la ideología de rol } \\
\text { Acuerdo con la mujer que debe }\end{array}$}} \\
\hline & & & & \\
\hline en su rol de madre & - & $0,06(0,11)^{* *}$ & - & - \\
\hline \multicolumn{5}{|l|}{ Trabajo de la mujer si los niños } \\
\hline son pequeños & & - & - & - \\
\hline Constante & - & 1,75 & - & - \\
\hline R2 ajustada (n) & - & $0,01(601)$ & - & - \\
\hline
\end{tabular}

$H$ ipótesis de solidaridad interfamiliar

Ayuda de padres

Ayuda de suegros

Constante

R2 ajustada
$-0,08(0,05)$ n.s. $-0,04(0,04)$ n.s. $0,06(0,04)$ n.s. $-0,06(0,05)$ n.s. $0,08(0,06)$ n.s. $-0,02(0,05)$ n.s. $-0,01(0,05)$ n.s. $-0,01(0,06)$ n.s.
2,1
1,5
1,6
2,8
$0,00(237)$
$0,00(244)$
$0,00(247)$
$0,00(234)$

${ }^{* *} p<1 \% ; *_{p}<5 \% ;+p<10 \% ;$ n.S. $=$ no significativo.

N ota: aquellas variables para las que no se podía rechazar la hipótesis nula para el coeficiente de regre sión parcial B se han excluido del modelo, salvo en la hipótesis de solidaridad interfamiliar, donde se han mantenido para ilustrar la ausencia de relación. 
la implicación del hombre en unas tareas que, por lo demás, son las que en las nuevas familias urbanas más feminizadas están. Comoquiera que el estatus profesional está fuertemente relacionado con la duración de la jornada de trabajo, no ha de extrañar que la participación masculina sea mayor no sólo cuando se trabaja fuera del hogar, sino también cuando el trabajo es más absorbente.

Las características familiares 0 individuales que influyen en las pautas de atención cotidiana de los niños guardan relación, por el contrario, tanto con la sobrecarga relativa de las familias, como con el estatus social de los cónyuges, pero no con la diferencia de recursos de los cónyuges. Así, cuanto mayor es el nivel educativo o el estatus profesional de la mujer, mayor es la implicación del hombre en esta tarea; por otro lado, y, en contra de la hipótesis de la teoría de los recursos, que estos datos parecen en principio sostener, la implicación del hombre crece con su nivel de estudios y con su estatus profesional. Por ello, lo que estos datos evidencian es que la participación masculina no depende de los recursos relativos de los cónyuges sino de su estatus social. Pero no sólo el estatus social de pertenencia tiene influencia en esta dimensión general de atención a los hijos, las disponibilidades de tiempo de ambos padres también condicionan las pautas de atención a los hijos, así como la edad de éstos, pero en un sentido inverso a la pauta tradicional: cuanto más pequeños, más participan. La ideología del rol maternal también parece tener cierta importancia, de suerte que en las familias donde la mujer tiene una visión menos tradicional hay una mayor participación masculina. Esta influencia, sin embargo, se manifiesta más aparente que real, pues, por un lado, los dos indicadores utilizados para medir la ideología de rol no presentan resultados coherentes y, por otro, la varianza explicada por la ideología de rol es mínima (apenas un 1\%).

Las pautas de atención educativa y cuidado en caso de enfermedad, por el contrario, está más determinada por las disponibilidades temporales de los cónyuges que por su estatus social, si bien a mayor nivel educativo del hombre y a mayor nivel profesional de la mujer, se da una mayor implicación del hombre. Así, los indicadores que miden la disponibilidad temporal de los cónyuges explican un $13 \%$ de la variación en las pautas de atención socioeducativa, mientras que los indicadores de recursos de los cónyuges solamente explican un $4 \%$ y la diferencia de rentas un $2 \%$. N i la ideología, ni la densidad de la solidaridad interfamiliar (medida a través de la frecuencia de ayuda) tienen influencia al guna en cómo resuelven las familias la supervisión educativa y la atención en caso de enfermedad.

A pesar de que la solidaridad interfamiliar encuentra en la ayuda en las tareas relacionadas con la atención y el cuidado de los niños una de sus manifestaciones típicas, y ésta es relativamente frecuente (pues un 23\% de las encuestadas afirmó recibir ayuda con frecuencia de los padres), esta solidaridad no tiene un efecto tradicionalizador sobre las pautas de distribución de las tareas entre los cónyuges. La intervención de los abuelos, ya sean maternos o paternos, para resolver algunas de estas tareas no supone, por tanto, una vía para el descompromiso de los padres. De hecho, la media de participación masculina es mayor en las familias en las que se declara recibir ayuda de los padres 0 
suegros con mucha frecuencia que en otros casos en los que esta ayuda es menos frecuente ${ }^{11}$.

Los resultados derivados de la consideración conjunta de todas las variables explicativas no se muestra plenamente consistente con los resultados obtenidos del análisis de las distintas hipótesis explicativas independientemente consideradas, al menos para al gunas de las tareas.

En lo que al juego con los hijos se refiere, en principio algunas de las variables que miden la sobrecarga relativa de la familia son las que más explican las pautas diferenciales de atención y cuidado de los hijos, pero en realidad lo que el análisis mediante regresión múltiple nos dice es que la participación del hombre depende de la edad de los hijos y de la hora a la que vuelve a casa. En otros términos, los padres participan más en el juego con sus hijos cuando éstos son más pequeños que cuando son más mayores, tanto durante el fin de semana como entre semana, si bien en este caso su grado de implicación depende de su hora de llegada a casa.

La atención matinal, por el contrario, está plenamente condicionada por las exigencias temporales y laborales de los cónyuges. Si la mujer no trabaja fuera del hogar, no existe ninguna variable que explique la escasa variación de la realización exclusiva por parte de la mujer de este conjunto de tareas. EI $70 \%$ de las encuestadas que no trabajan fuera del hogar levantan y se ocupan de los niños hasta que van al colegio, así como los llevan o acompañan al centro escolar siempre (opción 1, mujer siempre). Cuando la mujer dispone de un trabajo extradoméstico, por el contrario, existe una mayor participación del hombre en estas tareas, no tanto en función de las cargas familiares como tales, como en función de las restricciones temporales de la mujer (las únicas que se han medido) y de su estatus profesional. Las mujeres con un estatus profesional más elevado y cuanto más larga sea su jornada laboral consiguen arrancar una mayor implicación del hombre por las mañanas que las mujeres con menor cualificación profesional. No obstante, incluso en estos casos la participación masculina es muy escasa, pues un $50 \%$ de las encuestadas que trabajan fuera del hogar realizan siempre estas tareas en exclusiva.

En relación con la atención cotidiana, tampoco existe una explicación plenamente satisfactoria de ninguna de las hipótesis al ternativas planteadas. LoS resultados que se obtienen de la consideración conjunta de todas las variables potencialmente explicativas configura una imagen en la que la participación masculina en esta dimensión depende del nivel educativo del hombre y de la mujer, condicionado por su disponibilidad de tiempo, así como de la edad del

11. Así, la media de participación masculina en el conjunto de tareas denominadas satención cotidiana» cuando se recibe ayuda de los padres (de la mujer) con mucha frecuencia para realizar estas mismas tareas es de 2,04 (donde el valor 1 representa participación masculina nula y el valor 4 participación femenina nula), mientras que si sólo se recibe ayuda en casos muy particulares la media de participación es de 1,85. Valores prácticamente idénticos se obtie nen si la ayuda recibida es de los suegros (de la mujer) (2,03 frente a 1,90). Si en lugar de considerar únicamente las tareas de atención cotidiana se consideran todas las demás tareas (salvo juego), no existe diferencia alguna (el val or medio del indicador es 1,7 en ambos casos). 
hijo menor, sin que ninguno de estos condicionantes sea claramente dominante sobre los demás. Aś, la implicación del hombre en la atención y el cuidado de sus hijos es mayor cuando la mujer trabaja también fuera del hogar a jornada completa y cuanto mayor es su nivel educativo o el del hombre y menor cuanto más tarde llega a casa y cuanto mayor es la edad del hijo menor. La imagen que se perfila, por tanto, es la de una implicación que depende del estatus social de los cónyuges, pero fuertemente condicionada por las disponibilidades temporales del marido y de forma menos directa de la mujer. Estas circunstancias sugieren que dicha implicación está definida en el espacio familiar en función de criterios básicamente culturales, según la norma: cuando la mujer trabaja fuera del hogar a tiempo completo, el hombre no sólo tiene que jugar con sus hijos, sino también cuidarlos y atenderlos, al menos mientras no sean lo suficientemente independientes como para hacerlo ellos mismos. Esta norma es válida también entre los estratos medios independientemente del estatus laboral de la mujer. La relación de la ideología de rol que se había detectado en el análisis separado de las distintas hipótesis alternativas, se evidencia como espúrea y desaparece una vez que se controla su efecto por la influencia de las variables real mente explicativas.

La participación en tareas relacionadas con la salud y la educación de los hijos, si bien está algo menos extendida que la atención cotidiana (media de

Tabla 4. M odelo final de participación masculina en las tareas de atención y cuidado de los niños. Coeficientes de regresión parcial sin estandarizar (estandarizados). A. Atención matinal; B. Atención cotidiana; C. Atención educativo-sanitaria; D. Juegos.

\begin{tabular}{|c|c|c|c|c|}
\hline Variables & A & B & C & D \\
\hline Nivel educativo del hombre & - & $0,06(0,12)+$ & $0,09(0,18)^{* *}$ & - \\
\hline $\mathrm{N}$ ivel educativo de la mujer & 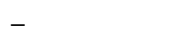 & $0,07(0,11)+$ & r & - \\
\hline Nivel profesional de la mujer & $0,14(0,19)^{* *}$ & $0,1(0,16)^{* *}$ & $0,05(0,09)^{*}$ & $0,09(0,12)^{*}$ \\
\hline $\begin{array}{l}\text { D uración de la jornada laboral } \\
\text { (base no trabaja): }\end{array}$ & & & & \\
\hline$M$ enos de 10 horas & $-0,15(-0,04)$ & $0,02(0,01)$ & $0,09(0,03)$ & - \\
\hline De 10 a 30 horas & 0 , & & + & - \\
\hline De 31 a 40 horas & ** & $0,40(($ & $0,33(0,19)^{* *}$ & - \\
\hline M ás de 40 horas & & $0,22(0,08)$ & $0,38(0,15)^{* *}$ & - \\
\hline Edad del hijo menor & - & $-0,02(-0,12)^{*}$ & - & - \\
\hline $\begin{array}{c}\text { Ciclo familiar (base } 0 \text { a 3) } \\
\text { Edad mavor de } 4 \text { a } 5\end{array}$ & & & & \\
\hline $\begin{array}{l}\text { Edad mayor de } 4 \text { a } 5 \\
\text { Edad mavor de } 6 \text { a } 13\end{array}$ & & & $0,20(0,10)+$ & $-0,02(-0,01)$ \\
\hline $\begin{array}{l}\text { Edad mayor de } 6 \text { a } 13 \\
\text { Edad mayor de } 13 \text { a } 18\end{array}$ & & & $\begin{array}{l}0,40( \\
0,44(\end{array}$ & $\begin{array}{l}-0,10(-0,06) \\
-0,30(-0,14)^{*}\end{array}$ \\
\hline Edad $\mathrm{r}$ & & & $0,37($ & $-0,32(-0,11)^{*}$ \\
\hline H ora llegada hombre a casa & - & $-0,08(-0,14)^{* *}$ & $-0,14(-0,25)^{* *}$ & $-0,10(-0,14)^{* *}$ \\
\hline Constante & 1,1 & 1,78 & & \\
\hline $\begin{array}{l}\text { R2 ajustada ( } n \text { ) } \\
\text { M edjia de narticinación masculina }\end{array}$ & & 0,1 & $0,18(404)$ & $0,03(404)$ \\
\hline (desviación estándar) & $1,5(0,82)$ & $1,9(0,71)$ & $1,7(0,63)$ & $2,65(0,81)$ \\
\hline
\end{tabular}

${ }^{* *} p<1 \%$; ${ }^{*}<5 \%$; $+p<10 \%$; en los demás casos $p>10 \%$, por lo que se puede descartar la hipótesis nula para el coeficiente de regresión parcial $B$. 
participación 1,7 frente a 2,0), depende básicamente del mismo tipo de variables, aunque no en todos los casos en la misma dirección. Los padres se implican más en la ayuda con los deberes cuanto mayor es su nivel educativo y a mayor edad de los hijos, aunque fuertemente condicionado por sus limitaciones temporales e ind ependientemente del estatus laboral de la mujer. El acompañamiento al médico también es más frecuente que lo realice el padre de forma conjunta o alternativa con la madre cuanto mayor es su nivel educativo, si la mujer trabaja fuera del hogar y dependiendo de sus disponibilidades temporales, pero independientemente de la edad de los hijos. La imagen que así se perfila es la de una participación masculina en el cuidado de la salud y en la educación de sus hijos condicionada al estatus social de pertenencia de los padres, pero supeditado a las limitaciones temporales de los cónyuges.

\section{Resumen y conclusiones}

Las tareas relacionadas con la atención y el cuidado de los hijos siguen siendo mayormente realizadas por las mujeres. Ahora bien, la participación masculina en la resolución de dichas tareas ha aumentado durante la última década, al menos entre las nuevas familias urbanas. El ritmo de implicación del hombre en el cuidado de sus hijos ha sido, no obstante, bastante limitado; de hecho, bastante menor que el que se ha producido en el ámbito de las tareas domésticas. En conjunto, sin embargo, puede afirmarse que entre estas familias se está registrando un proceso no muy rápido pero continuo de desdiferenciación de los roles conyugales y domésticos.

La implicación masculina en la atención y el cuidado de los hijos está fuertemente condicionada por el tipo de actividades de las que se trata. M ás allá de la tradicional mayor participación en el juego, la hora a la que se realiza la tarea en concreto, así como el tipo de tarea de que se trata, influye de forma decisiva en la frecuencia de participación masculina. Por ello, la consideración de las actividades y tareas relacionadas con los niños como una sola tarea familiar, como habitualmente suele hacerse en las encuestas, no solo no hace justicia a la realidad familiar, sino que además produce resultados claramente distorsionados.

Por otro lado, no existe ninguna teoría microsociológica que consiga dar cuenta adecuadamente de las distintas dimensiones del cuidado y la atención de los hijos. La vida familiar es una realidad multifacética fuertemente centrada en la mujer y donde la participación masculina depende del tipo de actividad, de la sobrecarga relativa de la familia (condicionada por la edad de los hijos y las disponibilidades horarias de ambos padres) y del estatus social, fundamentalmente educativo, de los padres. La ideología de rol familiar ${ }^{12}$ de la

12. La ideología política medida a través de una escala convencional de siete puntos entre extrema izquierda y extrema derecha tampoco influye en las pautas de atención y cuidado de los hijos. En este sentido, las familias de derechas no son ni más ni menos participativas que las familias de izquierdas. 
madre no influye significativamente en las pautas de participación del hombre. La densidad de las relaciones con la parentela, medida a través de la frecuencia de la ayuda recibida, tampoco condiciona el carácter más o menos tradicional de los roles parentales.

La creciente incorporación de la mujer al mercado de trabajo y los niveles de escolarización cada vez más el evados que se están registrando en los núcleos urbanos en la actualidad deberían haber redundado en una mayor implicación del hombre en la atención y el cuidado de los hijos. El aumento de la partici-

Tabla 5. Evolución de la división del trabajo doméstico en M adrid, 1984-1995. Porcentaje de familias en las que las distintas tareas se realizan bien por la mujer (siempre o la mayor parte de las veces), por el hombre (siempre o la mayor parte de las veces) o conjuntamente.

\begin{tabular}{|c|c|c|c|c|c|c|c|c|}
\hline \multirow[b]{2}{*}{ Tareas } & \multicolumn{2}{|c|}{ M ujer } & \multicolumn{2}{|c|}{ Conjuntam. } & \multicolumn{2}{|c|}{ Hombre } & \multicolumn{2}{|c|}{ Otros } \\
\hline & 1984 & 1995 & 1984 & 1995 & 1984 & 1995 & 1984 & 1995 \\
\hline Compra de todos & & & & & & & & \\
\hline los días & 91 & 73 & 6 & 19 & 1 & 4 & 8 & 3 \\
\hline $\begin{array}{l}\text { Compra de ropa } \\
\text { y calzado }\end{array}$ & & & & & & & & \\
\hline Compra de bienes & T & $J 2$ & 21 & & 2 & 1 & J & 2 \\
\hline duraderos & 21 & 16 & 64 & 81 & 5 & 2 & 10 & 1 \\
\hline $\begin{array}{l}\text { Preparar desayuno y } \\
\text { meriendas }\end{array}$ & 85 & 67 & 3 & 24 & 1 & 4 & 10 & 4 \\
\hline Preparar comida y cena & 91 & 68 & 3 & 19 & 0 & 1 & 3 & 1 \\
\hline Poner y recoger la mesa & 69 & 42 & 8 & 31 & 3 & 8 & 19 & 17 \\
\hline Fregar platos & 87 & 67 & 3 & 23 & 1 & 3 & 8 & 5 \\
\hline Lavar y planchar la ropa & 87 & 88 & 2 & 8 & 1 & 0 & 8 & 2 \\
\hline Limpieza de la casa & 85 & 73 & 3 & 19 & 1 & 1 & 11 & 7 \\
\hline Recoger la ropa sucia & 88 & 64 & 2 & 22 & 1 & 1 & 8 & 11 \\
\hline $\mathrm{H}$ acer las camas & - & 66 & - & 19 & - & 2 & - & 10 \\
\hline Recoger las habitaciones & $5-$ & 70 & - & 17 & - & 1 & - & 11 \\
\hline Limpieza de zapatos & 62 & 47 & 9 & 25 & 3 & 2 & 25 & 13 \\
\hline Tareas de costura & 93 & 92 & 0 & 1 & 1 & 0 & 6 & 6 \\
\hline Resolver los asuntos & & & & & & & & \\
\hline con el banco & 29 & 37 & 35 & 36 & 19 & 26 & 16 & 1 \\
\hline Cuidar del buen funcio- & & & & & & & & \\
\hline namiento del coche & 7 & 2 & 6 & 8 & 36 & 78 & 50 & 8 \\
\hline $\begin{array}{l}\text { Atención a parientes } \\
\text { enfermos }\end{array}$ & 8 & 31 & & & 3 & 2 & 27 & te \\
\hline EmIn & 0 & $J_{\perp}$ & 11 & Ju & J & $J$ & 21 & 10 \\
\hline
\end{tabular}

Fuente: Los datos de 1984 corresponden a los resultados para la Comunidad Autónoma de M adrid de la encuesta 1431 del CIS. Los datos correspondientes a 1995 proceden de la «encuesta a nuevas familias en nuevos municipios» realizada por el equipo de investigación dirigido por Gerardo Meil y formado por los profesores Luis E. Alonso y Lourdes Pérez (UAM ), con la colaboración de los estudiantes de 4ํ y 50 curso de la especialidad de Sociología Económica de la licenciatura en Economía de la U AM . 
pación que se ha registrado en la última década no se corresponde, sin embargo, con la envergadura de dichos cambios. La emergencia de un modelo de paternidad como el preconizado por Bill Cosby en la serie televisiva, donde no sólo se afirma que los hijos son lo más importante de la vida, sino que se dedica tiempo y energía a satiffacer sus necesidades, no constituye un modelo en rápida difusión entre los españoles (a juzgar por las respuestas dadas por las madres).

\section{Bibliografía}

BarnetT, R.C.; BARUCH, G. (1987). «D eterminants of Fathers' Participation in Family Work». Journal of $M$ arriage and the Family, 49, p. 29-40.

BIRD, G.; SCRUGGS, M . (1984). «D eterminants of Family Task Sharing: A Study of $H$ usbands and W ives». Journal of M arriage and the Family, mayo, p. 345-355.

BLAIR, S.L.; LICHTER, D. (1991). «M easuring the Division of H ousehold Labor». Journal of Family Issues, 12, p. 91-113.

BotT, E. (1971). Family and social network. Londres: Tavistock (hay traducción castellana en Taurus).

Centro de In Vestigaciones So ciológicas (1984). Estudio núm. 1433, sobre «D esigualdad familiar y doméstica». M adrid: CIS.

COVERM AN , S. (1985). «Explaining H usband's Participation in D omestic Labor». The Sociological Q uarterly, vol. 26, núm. 1, p. 81-97.

DURÁN, M a Ángeles (1988). De puertas adentro. M adrid: Instituto de la M ujer.

EN GLAN D, F.; FARKAS, G . (1986). H ouseholds, Employment and Gender. N ueva York: Aldine Press.

H EGNER, F.; LACKERM AN N , U . (1989). «Familienhaushalt und Erwerbstätigkeit». N AVE-H ERZ, R.; M ARKEFKA, M. (ed.). H andbuch der Familienforschung. Bd.1: Familienforschung, Frankfurt: Luchterhand, p. 491-512.

H Ö PFLIN GER, F.; C H ARLES, M . (1990). «Innerfamiliale Arbeitsteilung: mikrosoziologische Erklärungsansätze und empirische Beobachtung». Zeitschrift für Familienforschung, 2, p. 87-113.

Keddi, B.; SeIden SPIn ner, G. (1991). «Arbeitsteilung und Partnerschaft». Bertram, $H$. de. D ie Familie in Westdeutschland. O pladen: Leske + Budrich, p. 159-192.

Kellerhals, J.; Perrin, J.F.; Steinauer-Cresson, G.; Voneche, L.; Wirth, G. (1982). M ariages au quotidien. Lausanne: P.M . Favre.

K O O PM AN-BOYDEN , P.; ABвоT T, M. (1985). «Expectations for H ousehold Task Allocation and Actual Task Allocation: A N ew Zealand Study». Journal of M arriage and the Family, febrero, p. 211-220.

LESLIE; AN DERSON ; BRAN SO N (1991). «Responsability for children». Journal of Family Issues, 12, 2, p. 197-210.

LEWIS-BECK, de (1993). Regression-Analysis. Londres: Sage.

M ARET, E.; FIN LAY, B. (1984). «T he D istribution of H ousehold Labor Among Women in D ual-earner Families». Journal of M arriage and the Family, mayo de 1984, p. 357-364.

M EIL LANDWERLIN, G . (1996). «La redefinición de la división del trabajo doméstico en la nueva familia urbana» (en prensa).

M In IStère des Affaires So ciaux (1988). Encuesta del M inistère des Affaires SociauX.

En FO RSÉ, M. y otros. Recent Social Trends in France, enero de 1988, p. 1960 1990, Campus Verlag, 1993. 
M In ISterio de Asuntos So ciales (1994). Relaciones padres-hijos. M adrid (investigación dirigida por $\mathrm{F}$. $\mathrm{O}$ rizo).

N AVE-H ERZ (1989). «Zeitgeschichtlicher Bedeutungswandel von Ehe und Familie in der Bundesrepublik D eutschland». En N AVE-H ERZ, R.; M ARKEFKA, M . (ed.). $\mathrm{H}$ andbuch der Familienforschung. Bd.1: Familienforschung. Frankfurt: Luchterhand, p. 211-222.

O AKLEY, A. (1974). The Sociology of H ousework. Londres: M . Robertson.

ReXroat, C.; SHeH AN, C. (1987). «T he family life cycle and spouses' time in housework». Journal of $M$ arriage and the Family, 49, p. 737-750.

Ro BIN SON, J. (1977). H ow American U se Time. N ueva York: Praeger.

Ross, C.E. (1987). «T he D ivision of Labor at H ome». Social Forces, 65, p. 816-833. 